Where's the wine? Heavy social drinkers show attentional bias towards alcohol in a visual conjunction search task.

Charlotte R. Pennington ${ }^{1 *}$, Daniel J. Shaw ${ }^{1}$, Jennifer Adams ${ }^{2}$, Phoebe Kavanagh ${ }^{2}$, Holly Reed $^{2}$, Madeleine Robinson ${ }^{2}$, Emily Shave ${ }^{2}$, Hollie White ${ }^{2}$

${ }^{1}$ Department of Psychology, School of Life \& Health Sciences, Aston University, Birmingham, B4 7ET.

${ }^{2}$ Department of Health and Social Sciences, Faculty of Health and Applied Sciences, University of the West of England, Coldharbour Lane, Bristol, BS16 1QY.

*Corresponding author: Charlotte R. Pennington, E-mail: charlotte.pennington@ outlook.com

Abstract: 299 words

Main Body: 3993 words (new method described)

Figures: 2

Tables: 4

Supporting Information Files: 2

Data Availability Statement: The pre-registration protocol, data and supporting information files for this study are available via the Open Science Framework (OSF): https://osf.io/46sn8/

Funding: None.

Conflict of Interest: None. 


\begin{abstract}
Background and Aims: Research indicates that high consumers of alcohol exhibit attentional bias $(\mathrm{AB})$ towards alcohol-related cues, suggestive of a cognitive mechanism that might drive substance seeking. Many tasks that measure AB (e.g., visual probe, addiction Stroop), however, are limited by their reliance on non-appetitive control cues, the serial presentation of stimuli, and their poor internal reliability. The current study employed a visual conjunction search (VCS) task capable of presenting multiple alcoholic and non-alcoholic appetitive cues simultaneously to assess whether social drinkers attend selectively to alcoholic stimuli. To assess the construct validity of this task, we examined whether alcohol consumption and related problems, subjective craving, and drinking motives predict alcohol-specific AB. Design \&
\end{abstract} Setting: A VCS task was performed in a laboratory setting, which required participants to detect the presence of appetitive alcoholic (wine, beer) and non-alcoholic (cola, lemonade) targets within arrays of matching and non-matching distractors. Participants: Data from 99 participants were assessed $\left(M_{\text {Age }}=20.77, S D=2.98 ; 64\right.$ [65\%] females $)$, with $81.8 \%$ meeting the threshold for harmful alcohol consumption $\left(M_{\text {AUdIT }}=12.89, S D=5.79\right)$. Measurements: Self-reports of alcohol consumption and related problems (AUDIT), subjective craving (Alcohol Craving Questionnaire Short Form) and drinking motives (Drinking Motives Questionnaire Short Form) were obtained, and the VCS task measured response times for the correct detection of alcoholic and non-alcoholic targets. Findings: Participants were significantly quicker to detect alcoholic relative to non-alcoholic appetitive targets $(p<.001$, $\left.d_{z}=.41\right)$, which was predicted positively by AUDIT scores $\left(p=.013, R^{2}=.06 \%\right)$. The VCS task achieved excellent reliability $(\alpha>.79)$, superior to other paradigms. Conclusions: The Visual Conjunction Search task presents as a highly reliable method for assessing alcoholrelated attentional bias, and shows that heavy social drinkers prioritise alcoholic cues in their immediate environment.

Key words: Alcohol consumption; AUDIT; attentional bias; visual search; visual attention; subjective craving; drinking motives. 
ALCOHOL-RELATED ATTENTIONAL BIAS

\section{Where's the wine? Heavy social drinkers show attentional bias towards alcohol in a visual conjunction search task.}

A vast corpus of research indicates that heavy social drinkers allocate their attention selectively towards alcohol-related cues (see 1 for review). This attentional bias (AB) towards alcohol is believed to reflect enhanced neural signalling for cues associated with substances administered repeatedly, and the degree to which an individual exhibits alcohol-related $A B$ is suggested to be an important predictor of substance seeking and relapse risk (2). In this light, it is essential to develop tools for the accurate and reliable measurement of AB; only then can we begin to advance our understanding of the relationship between alcohol-related $A B$ and harmful alcohol consumption and assess the effectiveness of interventions.

Alcohol-related $\mathrm{AB}$ has been demonstrated with a variety of experimental paradigms, including the addiction Stroop (3-8), visual probe (9-12), anti-saccade $(13,14)$ and gaze contingency task $(15,16)$. On the visual probe task, alcohol users respond faster to probes appearing in locations occupied previously by alcohol-related compared to non-alcoholic stimuli, suggesting their attention is drawn to the spatial location of alcoholic cues (1). Moreover, $\mathrm{AB}$ on this task appears to be moderated by alcohol use, with heavy social drinkers demonstrating greater alcohol-related $\mathrm{AB}$ compared with lighter drinkers $(12,17)$. Similarly, studies employing the addiction Stroop task report that heavy drinkers and alcohol-dependent individuals are slower to name the colour in which alcohol-related words are presented relative to control words $(4,6-8,18-20)$. The findings of studies employing these two tasks therefore converge to suggest that individuals who consume alcohol regularly demonstrate preferential processing of alcohol-related stimuli.

In recent years, however, both the visual probe and addiction Stroop task have come under increasing criticism for their low internal and test-retest reliability (21-23). It has also 
ALCOHOL-RELATED ATTENTIONAL BIAS

been argued that the type of stimuli used in experimental paradigms may exaggerate the degree to which AB towards alcohol is shown (24). Some studies, for example, assess alcohol-related $\mathrm{AB}$ by comparing responses between alcoholic appetitive stimuli and non-alcoholic nonappetitive control stimuli (e.g., office stationary; 13,16,25-28). By only using non-appetitive control cues, it is impossible to dissociate between alcohol-specific $\mathrm{AB}$ or a more generalised response bias towards appetitive stimuli imbued with incentive value (see 1). Indeed, a number of studies have demonstrated the importance of using appetitive non-alcoholic control stimuli to investigate alcohol-related cognitions (14,24,29-32). Finally, experimental measures of AB towards alcoholic cues often present participants with (non-)alcoholic stimuli in a sequential manner that fails to capture an important aspect of real-world contexts: In naturalistic environments (e.g., pubs and bars), multiple contrasting cues will place demands on visual attention simultaneously. In the addiction Stroop and anti-saccade tasks, however, individuals respond to single items presented successively, and in the visual probe task they respond to a probe after the presentation of a singular pair of alcoholic and control stimuli. It remains to be seen, then, whether alcohol users demonstrate $A B$ towards alcohol even when multiple nonalcoholic appetitive distractors are present.

These methodological limitations are overcome with visual search paradigms that afford the presentation of multiple matched alcoholic and non-alcoholic appetitive cues. The visual conjunction search (VCS) task requires individuals to scan a stimulus array for a prespecified target positioned among multiple matching and non-matching distractor images. In comparison to feature searches, in which targets appear to "pop out" by virtue of a single distinguishing characteristic, detection of the target in conjunction search demands more attentional resources because it shares one or more common features with the distractors. Given this high visual similarity, participants must use prior knowledge of target characteristics to maximise search efficiency (33-35). This task may therefore offer an effective method for 
ALCOHOL-RELATED ATTENTIONAL BIAS

assessing alcohol-related $\mathrm{AB}$ and substance-related $\mathrm{AB}$ more generally; through repeated alcohol use, characteristic features of alcoholic cues may elicit enhanced signalling that leads to their prioritised detection among other stimuli in the environment (24). This aligns with the incentive-sensitisation theory of addiction (36-38), which proposes that, through neurophysiological changes, increased familiarity with substance-related cues heightens their incentive motivational properties $(1,39,40)$. Despite their potential utility, we are aware of only one prior use of the VCS task to assess alcohol-related AB. In a previous experiment (24), we asked social drinkers to identify either an alcoholic (beer), appetitive non-alcoholic (water) or non-appetitive target (detergent) in an array of matching and mismatching distractors. Participants exhibited faster responses to both alcoholic and non-alcoholic relative to nonappetitive targets. At first glance, this appears to reveal generalised AB towards appetitive stimuli. However, it is equally possible that by pairing appetitive non-alcoholic targets with non-appetitive repellent distractors in the same scene, we inadvertently masked alcohol-related $\mathrm{AB}$ with an approach bias towards palatable non-alcoholic stimuli.

Overcoming the aforementioned issues of previous research, the first aim of the current study was to examine whether alcohol users exhibit $\mathrm{AB}$ towards alcoholic (e.g., wine) relative to matched appetitive non-alcoholic stimuli (e.g., cola) using a novel VCS task. It was hypothesised that social drinkers would exhibit greater $\mathrm{AB}$ towards alcohol relative to nonalcohol, indexed by faster reaction times to detect the presence of alcoholic targets. To assess the construct validity of this task, the second aim was to examine whether self-reported alcohol consumption and related problems, subjective craving and drinking motives predicted alcoholrelated $\mathrm{AB}$. Previous research suggests that the degree to which individuals demonstrate alcohol-related $\mathrm{AB}$ is proportional to the amount of alcohol they consume habitually (e.g., 4,12,41-43). Similarly, studies report a reliable association between subjective craving and $\mathrm{AB}$, whereby heightened craving leads to attentional capture by associated cues until the 
ALCOHOL-RELATED ATTENTIONAL BIAS

substance is consumed (e.g., 10,44-46). Drinking motives refer to the particular valued outcomes that people associate with drinking (e.g., social, enhancement, coping, conformity; $47,48)$, and studies indicate that these serve as a unique predictor of drinking behaviour; whilst coping motives are related to alcohol-related consequences (e.g., impaired control), social and enhancement motives are related to higher levels of consumption (49-54). Driven by this research, we hypothesised that alcohol consumption and related problems (AUDIT), subjective craving and drinking motives would each predict alcohol-related $\mathrm{AB}$ positively and independently.

\section{Method}

\section{Data Availability Statement}

The pre-registration protocol, data, and supporting information files are available via https://osf.io/46sn8/.

\section{Design}

Participants completed a visual conjunction search (VCS) task comprising a 2 (Target: Alcoholic vs. Non-alcoholic) x 2 (Presence: Present vs. Absent) within-participant design, and three self-report questionnaires. An a-priori power analysis ( $\mathrm{G}^{*}$ Power; 55) indicated that a sample size of 88 was required to detect a moderate main effect of Target (Cohen's $d=.50, d_{z}$ $=.35)$ with $90 \%$ power at alpha $=.05$. However, a minimum sample of 100 participants was planned in order to allow students to collect course credits by the end of the university term and to ensure we met guidelines proposed for regression analyses (i.e., $50+8 k$; 56,57).

\section{Participants}


ALCOHOL-RELATED ATTENTIONAL BIAS

Data from a total of 129 participants were collected because, unexpectedly, 27 participants were excluded for achieving less than 50\% accuracy on the VCS task. Given that accuracy is typically very high on this task $(33,35)$, such poor performance indicates a misunderstanding of instructions or lack of concentration. Additionally, two participants were excluded because they reported not drinking alcohol (i.e. AUDIT score of zero) and one responded inconsistently. Importantly, these exclusions do not alter the reported findings (see File S1). The final sample included 99 participants (64 [65\%] females) aged 18-45 years $($ Mage $=20.77, S D=2.98)$, all of whom reported normal or corrected-to-normal vision. With a mean AUDIT score of 12.89 $(S D=5.79$, range 3-30), and 81.8\% meeting the threshold for harmful alcohol use (score > 8; 58), this sample was comprised predominantly of heavy social drinkers.

Ethical approval was granted by the Faculty Research Ethics Committee and all individuals provided informed consent prior to commencing the experiment.

\section{Measures}

The VCS task and self-report questionnaires were administered through the Cogent toolbox for MATLAB (MathsWorks, Natick, MA).

\section{Visual Conjunction Search Task}

The VCS task required participants to detect whether a left-hanging target (alcoholic or nonalcoholic) was present or absent within an array of alcoholic and non-alcoholic distractors. Four images of branded alcoholic (beer or red wine) and non-alcoholic beverages (lemonade or cola) were selected from the Amsterdam Beverage Picture Set (59) - a validated battery of alcoholic and non-alcoholic stimuli. These stimuli were matched according to the normative ratings reported by Pronk et al. (59) based on context and sub-content (single bottles with no contextual cues) and product familiarity (> 90\%; "Do you know this beverage?" and "Have 
ALCOHOL-RELATED ATTENTIONAL BIAS

you ever consumed this beverage?" [Yes/No]). Search arrays were then created comprising alcoholic and non-alcoholic stimuli that were equivalent in terms of their dominant colour and luminosity. In arrays containing alcoholic targets, participants were instructed to detect the presence/absence of a left-hanging green beer bottle amongst distractors of matching beer bottles and mismatching green lemonade bottles; or a red wine bottle amongst an array of red cola distractors. These instructions were reversed for the non-alcohol target trials. Stimulus arrays were displayed on an invisible 4 x 6 grid containing 24 items. The target and half of the non-matching distractor images were rotated 45 degrees to the left (see Figure 1).

\section{[INSERT FIGURE 1 HERE]}

In line with Biggs, Clark and Mitroff (60), there were four blocks of 32 trials. The same trial type was presented throughout each block, defined by the target-distractor combination (wine, beer, cola, lemonade). The order of the 32 trials were randomised within blocks, with the target present on $50 \%$ of trials. Trials began with a black fixation cross presented for $500 \mathrm{~ms}$, after which the search array was displayed until a response was recorded. Participants responded to each trial by clicking directly on the target location with the computer mouse when it was present, and clicking anywhere within the black background surrounding the array when it was absent. The cursor reset to the centre of the computer screen at the beginning of each trial. Mouse clicks were coded according to their $x-y$ screen co-ordinates, and correct responses were recorded by predefined boundaries. The dependent variable was reaction time (RT) in seconds for correct responses, collapsed across the two alcoholic (wine and beer) and non-alcoholic target blocks (lemonade and cola). Quicker RT was used as an index of AB.

Further controls were implemented to ensure that search performance between the alcoholic and non-alcoholic stimuli was not confounded by target placement. Specifically, 
ALCOHOL-RELATED ATTENTIONAL BIAS

search arrays were designed such that the randomisation of alcoholic and non-alcoholic target positions were matched (e.g., in a trial where an alcoholic target appeared on the third column of the first row, there would be an exact match of this using a non-alcoholic target). The target was never presented on the first column of the first row, and each stimulus was presented equally on one of the four rows (i.e. in random order, each target appeared eight times in each row). To control for order effects, the task was counterbalanced so that each participant started on a block of alcoholic or non-alcoholic target trials.

\section{Questionnaires}

\section{Alcohol Consumption}

The Alcohol Use Disorders Identification Test (AUDIT; 61) is a 10-item screening tool used to assess harmful and hazardous alcohol consumption, drinking behaviours, and alcoholrelated problems. This questionnaire achieved acceptable internal reliability (McDonalds's $\omega$ $=.82$; Cronbach's $\alpha=.78$ ) and a total score ranging from 0-40 was calculated.

\section{Subjective Craving}

The Alcohol Craving Questionnaire Short Form (ACQ-SF-R; 62,63) includes 12-items that assess subjective craving. This also achieved acceptable internal reliability $(\omega=.86 ; \alpha=$ $.85)$ and a total score ranging from 12-84 was calculated.

\section{Drinking Motives}

The Drinking Motives Questionnaire Short Form (DMQ-R-SF; 47,64) includes 12items that assess participants' motivations for drinking alcohol through four sub-scales; social (positive social rewards), coping (dealing with negative emotions), enhancement (positive mood), and conformity (fitting in with peers). This questionnaire achieved high internal 
ALCOHOL-RELATED ATTENTIONAL BIAS

reliability when considering all items together $(\omega=.82 ; \alpha=.81)$, and those relating specifically to the Social $(\omega=.85 ; \alpha=.85)$, Coping $(\omega=.84 ; \alpha=.83)$, Enhancement $(\omega=.73 ; \alpha=.70)$ and Conformity sub-scales $(\omega=.77 ; \alpha=.77)$. The mean frequency for each motive was calculated separately with a possible range of 3-15.

\section{Procedure}

Participants always completed the AUDIT, ACQ-SF-R and DMQ-R-SF questionnaires before the VCS task, allowing us to examine if subjective craving predicted alcohol-related AB rather than the influence of alcohol-related images on subjective craving (65). The order of both blocks of the VCS task (alcohol or non-alcohol target blocks) and the questionnaires were counterbalanced between participants, however. Participants were seated $57 \mathrm{~cm}$ away from the computer monitor throughout the procedure. Upon completion of the study, participants received a full debrief.

\section{Analytic Strategy}

As expected, accuracy on the VCS task was high (>95\%) and so analyses focused on RT. To assess whether participants demonstrated $\mathrm{AB}$ towards alcohol, we conducted a 2 (Target: Alcoholic vs. Non-alcoholic) x 2 (Presence: Present vs. Absent) repeated measures Analysis of Variance (ANOVA) on RT. All main effects and interactions were analysed with Bonferroni corrections, and effect sizes are reported as partial eta squared and Cohen's $d_{z}$. Values present condition means $( \pm \mathrm{SD})$ and $95 \%$ confidence intervals for mean differences $\left(\mathrm{CI}_{\text {diff }}\right)$. Next, a hierarchical multiple regression analysis was conducted to examine whether alcohol consumption and related problems (AUDIT; step 1), subjective craving (ACQ-SF-R; step 2) and drinking motives (DMQ-R-SF; step 3) predicted alcohol-related AB. Here, a mean difference score was calculated by subtracting RT to alcoholic stimuli from non-alcoholic 
ALCOHOL-RELATED ATTENTIONAL BIAS

stimuli, with positive scores indicative of alcohol-related AB. Although predictor variables were correlated, variance inflation factors $(<1.50)$ and tolerance statistics suggested that multicollinearity was negligible. Furthermore, all predictors and outcomes met assumptions of normality. It is important to note that as a deviation from the pre-registration, the regression analysis entered the four sub-scales of the DMQ-R-SF separately (see File S1).

\section{Results}

Internal reliabilities for the VCS task are shown in Table 1. Using the Cocron application (66), we performed statistical comparisons between these estimates of internal reliability and those reported in previous research using the visual probe and addiction Stroop tasks $(21,23$; see File S2). This revealed that our VCS task significantly outperformed $69 \%$ of these former studies.

\section{[INSERT TABLE 1 HERE]}

Data from two participants on the VCS task were beyond 3 standard deviations of the sample mean and were replaced with the next lowest or highest score in the dataset (55). In line with predictions, there was a significant main effect of Target $(F[1,98]=18.00, p<.001$, $\left.\eta_{p}^{2}=.16\right)$, with participants quicker to identify alcoholic relative to non-alcoholic targets $(2.53$ $[ \pm .50]$ vs. $\left.2.68[ \pm .60] \mathrm{sec} ; \mathrm{CI}_{\text {diff }}=[-.23,-.08] ; p<.001, d_{z}=.41\right)$. There was also a significant main effect of Presence $\left(F[1,98]=263.16, p<.001, \eta_{\mathrm{p}}^{2}=.73\right)$, with participants quicker to detect the target when it was present relative to absent $\left(2.12[ \pm .30] v s .3 .09[ \pm .79] \mathrm{sec} ; \mathrm{CI}_{\text {diff }}=\right.$ $\left.[-1.09,-.85] ; p<.001, d_{z}=1.64\right)$. The two-way interaction between Target and Presence was inconclusive $(p>.05)$ and mirrored the main effects; response times were quicker for both present and absent alcoholic relative to non-alcoholic stimuli $(p s<.01)$, and quicker for present 
ALCOHOL-RELATED ATTENTIONAL BIAS

relative to absent stimuli $(p s<.001)$. One-sample $t$-tests on all dependent variables indicative of $\mathrm{AB}$ differed significantly from zero $(p s<.001)$. Table 2 presents descriptive statistics.

\section{[INSERT TABLE 2 HERE]}

The regression revealed that alcohol consumption and related problems (AUDIT) predicted alcohol-related $\mathrm{AB}$ positively $\left(\beta=.25 ; F[1,97]=6.47, p=.013, \mathrm{R}^{2}=.06 \%\right)$. This relationship is illustrated in Figure 2. Contrary to predictions, subjective craving and drinking motives did not account significantly for additional variance $(p s>.05)$. Table 3 presents the regression matrix.

[INSERT FIGURE 2 HERE]

[INSERT TABLE 3 HERE]

\section{Exploratory Analyses}

Given the results of our confirmatory analyses, we examined the relationships between alcohol consumption (AUDIT), subjective craving (ACQ-SF-R), drinking motives (DMQ-RSF) and alcohol-related AB independently of each other. Table 4 presents the correlation matrix. Interestingly, this revealed that alcohol use, subjective craving and social drinking motives each showed a weak but significant positive relationship with alcohol-related AB $(p<$ .028). In a data-driven approach, we then conducted separate regression analyses with alcohol consumption, subjective craving and social drinking motives entered as independent predictors of alcohol-related AB. This confirmed that each predictor significantly explained $4-5 \%$ of variance in alcohol-related $\mathrm{AB}$ exhibited on the VCS task. This suggests that each of these 
ALCOHOL-RELATED ATTENTIONAL BIAS

measures account for similar variance in $\mathrm{AB}$ towards alcohol; however, when entered in a hypothesis-driven hierarchical fashion, subjective craving and social drinking motives do not significantly explain additional variance in alcohol-related $\mathrm{AB}$ than alcohol consumption and related problems (AUDIT) do alone.

[INSERT TABLE 4 HERE]

\section{Discussion}

Uncovering the cognitive mechanisms that drive alcohol misuse is central to addiction research, facilitating the development of interventions capable of reducing such behaviour (for reviews see $1,2,40)$. In the present study, we evaluated the effectiveness of the visual conjunction search (VCS) task for measuring attentional bias (AB) towards alcohol - a cognitive mechanism believed to drive substance seeking and increase the risk of relapse (2). Our investigation demonstrated the high reliability and validity of this measure of alcohol-related $A B$; performance on the VCS task was itself predictive of, and associated with other known predictors of alcohol consumption. We argue, therefore, that this simple experimental task provides an accurate assessment of $\mathrm{AB}$ towards alcoholic cues, whilst also overcoming some fundamental methodological limitations of studies in this field.

Previous studies claim to demonstrate how alcohol users' visual attention is captured by alcohol-related cues through use of the addiction Stroop (3-8), visual probe (9-12), antisaccade $(13,14)$ and gaze contingency task $(15,16)$. Closer inspection of some of these investigations reveals fundamental methodological issues that may provide an inaccurate picture of alcohol-related cognition, however. Some demonstrations of alcohol-related AB have contrasted responses between alcoholic and non-alcoholic non-appetitive stimuli (e.g., 13,16,25-28). The use of such control stimuli renders it difficult to conclude that responses are 
ALCOHOL-RELATED ATTENTIONAL BIAS

due to the 'substance-relatedness' of stimuli rather than a more general appetitive response bias (for a related discussion see 1), and may lead to inflated estimates of the magnitude of alcoholrelated AB. Moreover, the addiction Stroop and visual probe task have been criticised for their poor reliability (21-23) and the sequential manner in which stimuli are presented $(24,30)$. We have shown that the VCS task achieves estimates of reliability that are markedly higher than these other tasks. Further, the simultaneous presentation of multiple alcoholic and nonalcoholic appetitive stimuli may capture how visual attention plays out in real-world environments more accurately. Thus, we contend that this paradigm proffers a more robust measurement of alcohol-specific $\mathrm{AB}$, and may prove useful in future studies that seek accurate assessment of $\mathrm{AB}$ towards other substances.

Building on the premise that repeat alcohol use may lead to enhanced signalling of alcohol-related cues (24), we hypothesised that alcohol users would exhibit AB towards alcoholic relative to non-alcoholic cues on the VCS task. Consistent with this, our sample of heavy social drinkers were quicker to detect the presence of alcoholic appetitive cues in an array of alcoholic and non-alcoholic distractors. These findings can be interpreted through the lenses of two theories: the incentive-sensitisation theory of addiction (36-38) and the more general feature integration theory of visual search $(33,34)$. The former suggests that through repeat consumption, alcohol-related cues acquire incentive-motivational properties that bias attentional processing towards alcoholic cues. The second suggests that visual search efficiency is enhanced through prior experience with, and knowledge of, target stimuli. Synthesising these accounts, our findings suggest that individuals who consume alcohol habitually may prioritise the detection of alcoholic stimuli in their immediate environment. These theories could be tested directly by personalising the stimuli used in the VCS task to reflect participants' beverage preference (see 67,68). If their primary tenets hold true, individuals who drink beer 
ALCOHOL-RELATED ATTENTIONAL BIAS

most regularly, for example, should exhibit greater $\mathrm{AB}$ towards this beverage relative to one that is less preferred.

At first glance, the findings of the present study seem to contrast with our previous experiment; using a similar VCS task, we observed generalised AB towards both alcoholic and non-alcoholic appetitive stimuli (24). It is important to highlight two key differences between these studies that may reconcile the discrepant findings. First, our previous design included a second control condition of non-appetitive stimuli (cleaning detergent) that may have heightened participants' approach bias towards non-alcoholic appetitive stimuli unintentionally, masking any subtle alcohol-specific AB. Second, our previous study involved a sample of light social drinkers with a mean AUDIT score of 6.5 and only $27 \%$ scoring above the clinical cut off of 8.0 (58). This differs strikingly from the sample of heavy social drinkers assessed in the present study; over $80 \%$ of the sample exceeded the threshold for harmful alcohol use, producing a mean AUDIT score of 12.9. Indeed, prior research indicates that heavy drinkers demonstrate greater alcohol-related $\mathrm{AB}$ than lighter drinkers $(12,17)$, so it is likely that these contrasting results reflect heterogeneity in participants' alcohol consumption and drink-related behaviours. Future research could investigate this by using our VCS task to compare alcohol-related $\mathrm{AB}$ between heavy and light drinkers, the latter of whom may not be so familiar with alcohol.

A second aim of the current study was to examine whether individual differences in alcohol consumption and related problems, subjective craving and drinking motives predicted $\mathrm{AB}$ towards alcohol. Findings indicated that AUDIT scores explained a small yet significant proportion of variability in alcohol-related AB. This serves to validate our task for the assessment of alcohol-related $\mathrm{AB}$, as well as providing further evidence for this association (see 4,12,41-43). Against predictions, however, subjective craving and drinking motives did not explain any unique variance in alcohol-related $\mathrm{AB}$. In a meta-analysis of 68 studies, Field 
ALCOHOL-RELATED ATTENTIONAL BIAS

et al. (40) report a weak but robust $(r=.19)$ positive relationship between craving and AB towards alcohol. Past research has also suggested that drinking motives are a unique predictor of alcohol consumption and related problems $(49,50,52-54)$. To probe this finding further, we conducted exploratory analyses to examine whether alcohol consumption, craving and social drinking motives predicted alcohol-related $\mathrm{AB}$ when they were entered into separate regression models. This indicated that each predictor explained between $4-5 \%$ of variance in alcoholrelated $\mathrm{AB}$ and showed moderate to strong correlations among themselves. However, when entered in a hierarchical fashion (as per confirmatory analyses), subjective craving and social drinking motives did not explain additional variance in alcohol-related AB than AUDIT scores alone. Further research should not be deterred from exploring these three potential predictors of alcohol-related $\mathrm{AB}$ more closely.

It is important to acknowledge some potential limitations of our experiment that can be addressed in future studies. First, to avoid any influence of alcohol-related images on measures of subjective craving (65), we administered all questionnaires before the VCS task. In doing so, however, we may have introduced the reverse order effect; self-reported craving may have influenced alcohol-related AB. This could be avoided easily by counterbalancing. Second, given the high proportion of students in the present sample who met the threshold for harmful alcohol use and related problems, future research should assess whether our findings extend to (non-student) samples reporting lower alcohol consumption or non-drinkers. Relatedly, further studies are needed to identify other sources of individual differences in AB towards alcohol; for example, while we have revealed that AUDIT scores are an important predictor, we did not consider other factors that might exert a confounding influence on this relationship (e.g., sociodemographic).

\section{Conclusion}


ALCOHOL-RELATED ATTENTIONAL BIAS

The current study presents the utility of the VCS task with carefully matched appetitive alcoholic and non-alcoholic stimuli for the assessment of $\mathrm{AB}$ towards alcoholic cues. We introduce a reliable and valid experimental measure of alcohol-related AB that captures more accurately how this cognitive process might influence visual attention in real-world contexts. Our findings serve as recommendations for future research in this area: First, it is paramount that researchers employ matched alcoholic and non-alcoholic stimuli if we are to conclude unequivocally that $\mathrm{AB}$ is driven by 'substance-relatedness'. Second, researchers must report more openly the reliability of experimental paradigms so that accurate inferences can be made regarding alcohol-related AB. Given the versatility of the VCS task, we welcome future studies to consider its use to uncover the attentional processes that may underpin substance (mis)use. 
ALCOHOL-RELATED ATTENTIONAL BIAS

\section{References}

1. Field, M., \& Cox WM. Attentional bias in addictive behaviors: A review of its development, causes, and consequences. Drug Alcohol Depend. 2008;(97):1-20.

2. Field, M., Marhe, R., \& Franken IHA. The clinical relevance of attentional bias in substance use disorders. CNS Spectr. 2014;(19):225-30.

3. Adams, S., Ataya, A. F., Attwood, A. S., \& Munafò MR. Effects of acute alcohol consumption on alcohol-related cognitive biases in light and heavy drinkers are taskdependent. J Psychopharmacol. 2011;(26):245-53.

4. Bruce, G., \& Jones BT. A pictorial Stroop paradigm reveals an alcohol attentional bias in heavier compared to lighter social drinkers. J Psychopharmacol. 2004;(18):527-33.

5. Cox, W. M., Fadardi, J. S., \& Pothos EM. The addiction-stroop test: Theoretical considerations and procedural recommendations. Psychol Bull. 2006;(132):443-76.

6. Field, M., Christiansen, P., Cole, J., \& Goudie A. Delay discounting and the alcohol Stroop in heavy drinking adolescents. Addiction. 2007;(102):579-86.

7. Hallgreen, K. A., \& McCrady BS. Interference in the alcohol Stroop task with college student binge drinkers. J Behav Heal. 2013;(2):112-9.

8. Sharma, D., Albery, I. P., \& Cook C. Selective attentional bias to alcohol related stimuli in problem drinkers and non-problem drinkers. Addiction. 2001;(96):285-95.

9. Fernie, G., Christiansen, P., Cole, J. C., Rose, A. K., \& Field M. Effects of 0.4g/kg alcohol on attentional bias and alcohol-seeking behaviour in heavy and moderate social drinkers. J Psychopharmacol. 2012;(26):1017-25.

10. Manchery, L., Yamush, D. E., Leuhring-Jones, P., \& Erblich J. Attentional bias to alcohol stimuli predicts elevated cue-induced craving in young adult social drinkers. Addict Behav. 2017;(70):14-7.

11. Miller, M. A., \& Fillmore MT. The effect of image complexity on attentional bias 
ALCOHOL-RELATED ATTENTIONAL BIAS

toward alcohol-related images in adult drinkers. Addiction. 2010;(105):883-90.

12. Townsend, J. M. \& Duka T. Attentional bias associated with alcohol cues: Differences between heavy and occasional social drinkers. Psychopharmacology (Berl). 2001;(157):67-74.

13. Jones, A., \& Field M. Alcohol-related and negatively valenced cues increase motor and oculomotor disinhibition in social drinkers. Exp Clin Psychopharmacol. 2015;(23):122-9.

14. Melaugh-McAteer, A., Curran, D., \& Hanna D. Alcohol attention bias in adolescent social drinkers: An eye tracking study. Psychopharmacology (Berl). 2015;(232):318391.

15. Qureshi, A., Monk, R. L., Pennington, C. R., Wilcockson, T. D. W., \& Heim D. Alcohol-related attentional bias in a gaze contingency task: Comparing appetitive and non-appetitive cues. Addict Behav. 2019;(90):312-7.

16. Wilcockson, T. D., \& Pothos EM. Measuring inhibitory processes for alcohol-related attentional biases: Introducing a novel attentional bias measure. Addict Behav. 2015;(44):88-93.

17. Field, M., Mogg, K., Zetteler, J., \& Bradley BP. Attentional biases for alcohol cues in heavy and light social drinkers: The role of initial orienting and maintained attention. Psychopharmacology (Berl). 2004;(174):88-93.

18. Cox, W. M., Brown, M. A., \& Rowlands LJ. The effects of alcohol cue exposure on non-dependent drinkers' attentional bias for alcohol-related stimuli. Alcohol Alcohol. 2003;(38):45-9.

19. Fadardi, J. S., \& Cox WM. Alcohol-attentional bias and motivational structure as independent predictors of social drinkers' alcohol consumption. Drug Alcohol Depend. 2008;(97):247-56. 
ALCOHOL-RELATED ATTENTIONAL BIAS

20. White, M. J., Cunningham, L. C., Pearce, R., \& Newnam S. Reward sensitivity predicts attentional bias towards alcohol-related cues in young binge drinkers. Personal Individ Differ. 2014;(60):13-4.

21. Ataya, A. F., Adams, S., Mullings, E., Cooper, R. M., Attwood, A. S., \& Munafò MR. Internal reliability of measures of substance-related cognitive bias. Drug Alcohol Depend. 2012;(121):148-51.

22. Field, M., \& Christiansen P. Commentary on Ataya et al. (2012). 'Internal reliability of measures of substance-related cognitive bias'. Drug Alcohol Depend. 2012;(124):18990.

23. Jones, A., Christiansen, P., \& Field M. Failed attempts to improve the reliability of the alcohol visual probe task following empirical recommendations. Psychol Addict Behav. 2018;(32):922-39.

24. Pennington, C. R., Qureshi, A. W., Monk, R. L., Greenwood, K., \& Heim D. Beer? Over here! Examining attentional bias towards alcoholic and appetitive stimuli in a visual search eye-tracking task. Psychopharmacology (Berl). 2019;1-12.

25. Brown, C. R. H., Duka, T., \& Forster S. Attentional capture by alcohol-related stimuli may be activated involuntarily by top-down search goals. Psychopharmacology (Berl). 2018;(235):2087-99.

26. Hicks, J. A., Fields, S., Davis, W. E. and Gable PA. Heavy drinking, impulsivity and attentional narrowing following alcohol cue exposure. Psychopharmacology (Berl). $2015 ;(232): 2773-9$.

27. Kvamme, T. L., Rømer Thomsen, K., Callesen, M. B., Doñamayor, N., Jensen, M., . . . Voon V et al. Distraction towards contextual alcohol cues and craving are associated with levels of alcohol use among youth. BMC Psychiatry. 2018;(18).

28. Kvamme, T. L., Pedersen, M. U., Overgaard, M., Rømer Thomsen, K., \& Voon V. 
ALCOHOL-RELATED ATTENTIONAL BIAS

Pupillary reactivity to alcohol cues as a predictive biomarker of alcohol relapse following treatment in a pilot study. Psychopharmacology (Berl). 2019;(236):1233-43.

29. Monk RL, Qureshi A, Pennington CR, Hamlin I. Generalised inhibitory impairment to appetitive cues: From alcoholic to non-alcoholic visual stimuli. Drug Alcohol Depend. 2017 Nov;

30. Pennington CR, Monk RL, Qureshi AW, Heim D. Alcohol Belongs Here: Assessing Alcohol-Related Inhibitory Control With a Contextual Go/No-Go Task. Exp Clin Psychopharmacol. 2019;

31. Ramirez, J. J., Monti, P. M. \& Colwill RM. Alcohol cue exposure effects on craving and attentional bias in underage college student drinkers. Psychol Addict Behav. 2015;(29):317-22.

32. Weafer, M. T., \& Fillmore J. Behavioral inhibition and addiction. In: In J. MacKillop \& H. de Wit (Eds.), editor. The Wiley-Blackwell handbook of addiction psychopharmacology. 2013. p. 135-64.

33. Treisman, A., \& Sato S. Conjunction search revisited. J Exp Psychol Hum Percept Perform. 1985;(114):285-310.

34. Treisman, A. M., \& Gelade G. A feature integration theory of attention. Cogn Psychol. 1980;(12):97-136.

35. Treisman, A \& Souther J. Search asymmetry: a diagnostic for preattentive processing of separable features. J Exp Psychol Gen. 1985;(114):285-310.

36. Berridge, K. C., \& Robinson TE. Liking, wanting, and the incentive-sensitization theory of addiction. Am Psychol. 2016;(71):670-9.

37. Robinson, T. E., \& Berridge KC. The neutral basis of drug craving: An incentivesensitization theory of addiction. Brain Res Rev. 1993;(18):247-91.

38. Robinson, T. E., \& Berridge KC. Addiction. Annu Rev Psychol. 2003;(54):25-53. 
ALCOHOL-RELATED ATTENTIONAL BIAS

39. Albery, I. P., Sharma, D., Noyce, S., Frings, D., \& Moss AC. Testing a frequency of exposure hypothesis in attentional bias for alcohol-related stimuli amongst social drinkers. Addict Behav Reports. 2015;(1):68-72.

40. Field, M., Munafò, M. R., \& Franken IH. A meta-analytic investigation of the relationship between attentional bias and subjective craving in substance use. Psychol Bull. 2009;(135):589-607.

41. Fadardi, J. S., \& Cox WM. Reversing the sequence: Reducing alcohol consumption by overcoming alcohol attentional bias. Drug Alcohol Depend. 2009;(101):137-45.

42. Field, M., Hogarth, L., Bleasdale, D., Wright, P., Fernie, G., \& Christiansen P. Alcohol expectancy moderates attentional bias for alcohol cues in light drinkers. Addiction. 2011;(106):1097-103.

43. Jones, A., Hogarth, L., \& Christiansen P. Reward expectancy promotes generalized increases in attentional bias for rewarding stimuli. Q J Exp Psychol. 2012;(65):233342.

44. Cox, W. M., Hogan, L. M., Kristian, M. R., \& Race JH. Alcohol attentional bias as a predictor of alcohol abusers' treatment outcome. Drug Alcohol Depend. $2002 ;(68): 237-43$.

45. Field, M., Mogg, K., Mann, B., Bennett, G. A., \& Bradley B. Attentional biases in abstinent alcoholics and their association with craving. Psychol Addict Behav. 2013;(27):71-80.

46. Franken IH. rug craving and addiction: Integrating psychological and neuropsychopharmacological approaches. Prog Neuro-Psychopharmacology Biol Psychiatry. 2003;(27):563-79.

47. Cooper ML. Motivations for alcohol use among adolescents: Development and validation of a four-factor model. Psychol Assess. 1994;(6):117-28. 
ALCOHOL-RELATED ATTENTIONAL BIAS

48. Cox, W. M., \& Klinger E. A motivational model of alcohol use. J Abnorm Psychol. 1988;(97):168-80.

49. Kuntsche, E., Gabainn, S. N., Roberts, C., Windlin, B., Vieno, A., . . Wicki M et al. Drinking motives and links to alcohol use in 13 European countries. J Stud Alcohol Drugs. 2014;(75):428-37.

50. Kuntsche, E., Knibbe, R., Gmel, G., \& Engels R. Why do young people drink? A review of drinking motives. Clin Psychol Rev. 2005;(25):841-61.

51. Magid, V., Maclean, M. G., \& Colder CR. Differentiating between sensation seeking and impulsivity through their mediated relations with alcohol use and problems. Addict Behav. 2007;(32):2046-61.

52. Merrill, J. E., \& Read JP. Motivation pathways to unique types of alcohol consequences. Psychol Addict Behav. 2010;(24):705-11.

53. Merrill, J. E., Wardell, J. D., \& Read JP. Drinking motives in the prospective prediction of unique alcohol-related consequences in college students. J Stud Alcohol Drugs. 2014;(75):93-102.

54. Read, J. P., Wood, M. D., Kahler, C. W., Maddock, J. E., \& Palfai TP. Examining the role of drinking motives in college student alcohol use and problems. Psychol Addict Behav. 2003;(17):13-23.

55. Faul, F., Erdfelder, E., Lang, A., \& Buchner, A. G*Power 3: A flexible statistical power analysis program for the social, behavioral, and biomedical sciences. Behav Res Methods. 2007; (39), 175-191.

56. Tabachnick, B. G., \& Fidell LS. Using multivariate statistics. (6th Ed.). Essex: Pearson Education Limited; 2014.

57. Wilson VanVoorhis, C. R., \& Morgan BL. Understanding power and rules of thumb for determining sample sizes. Tutor Quant Methods Psychol. 2007;(3):42-50. 
ALCOHOL-RELATED ATTENTIONAL BIAS

58. Babor, T. F., Higgins-Biddle, J. C., Saunders, J. B., \& Monteiro MG. The Alcohol Use Disorders Identification Test: Guidelines for use in primary care (2nd Ed.). Switzerland; 2001.

59. Pronk, T., van Deursen, D. S., Beraha, E. M., Larsen, H., \& Wiers RW. Validation of the Amsterdam Beverage Picture Set: A controlled picture set of cognitive bias measurement and modification paradigms. Alcohol Clin Exp Res. 2015;(39):2047-55.

60. Biggs, A. T., Clark, K., \& Mitroff SR. Who should be searching? Differences in personality can affect visual search accuracy. Personal Individ Differ. 2017;(116):3538.

61. Saunders, J. B., Aasland, O. G., Babor, T. F., De la Fuente, J. R. and Grant M. Development of the alcohol use disorders identification test (AUDIT). WHO collaborative project on early detection of persons with harmful alcohol consumptionII. Addiction. 1993;(88):791-804.

62. Singleton E. G., Tiffany, S. T., \& Henningfield JE. Development and validation of a new questionnaire to assess craving for alcohol. In: Proceedings of the 56th Annual Meeting, The College on Problems of Drug Dependence, Inc Volume II: Abstracts NIDA Research Monograph 153 Rockville, Maryland: National Institute on Drug Abuse, [Internet]. 1995. p. 289. Available from: http://adai.washington.edu/instruments/pdf/Alcohol_Craving_Questionnaire_Short_Fo rm_Revised_20.pdf

63. Singleton EG. Alcohol Craving Questionnaire, Short-Form (Revised; ACQ-SF-R): Background, Scoring, and Administration. Baltimore, MD, USA.; 1995.

64. Kuntsche, E., \& Kuntsche S. Development and validation of the Drinking Motive Questionnaire Revised Short Form (DMQ-R-SF). J Clin Child Adolesc Psychol. 2009;(38):899-908. 
ALCOHOL-RELATED ATTENTIONAL BIAS

65. Field, M., \& Eastwood B. Experimental manipulation of attentional bias increases the motivation to drink alcohol. Psychopharmacology (Berl). 2005;(183):350-7.

66. Diedenhofen, B., \& Musch J. Cocron: A web interface and R package for the statistical comparison of Cronbach's alpha coefficients. International Journal of Internet Science. 2016; (11): 51-60. Web tool available at: http://comparingcronbachalphas.org/

67. Christiansen P, Mansfield R, Duckworth J, Field M, Jones A. Internal reliability of the alcohol-related visual probe task is increased by utilising personalised stimuli and eyetracking. Drug Alcohol Depend. 2015;(155):170-4.

68. Houben, K., \& Wiers RW. Are drinkers implicitly positive about drinking alcohol? Personalizing the alcohol-IAT to reduce negative extrapersonal contamination. Alcohol Alcohol. 2007;(42):301-7. 
ALCOHOL-RELATED ATTENTIONAL BIAS

Table 1.

Internal reliability (McDonalds's $\omega$ and Cronbach's $\alpha$ ) for RT on the VCS task as a function of Target and Presence.

\begin{tabular}{lcccc}
\hline Target & \multicolumn{3}{c}{ Presence } \\
& \multicolumn{3}{c}{ Present } & \multicolumn{3}{c}{ Absent } \\
\hline & $\omega$ & $\alpha$ & $\omega$ & $\alpha$ \\
Alcoholic & 0.83 & 0.79 & 0.97 & 0.97 \\
Non-alcoholic & 0.89 & 0.82 & 0.97 & 0.97
\end{tabular}

Note: Each factor combination (e.g., Alcoholic-Present) consists of 32-items. 
ALCOHOL-RELATED ATTENTIONAL BIAS

Table 2.

Descriptive statistics for RT on the VCS task as a function of Target and Presence.

\section{Presence}

\begin{tabular}{lll}
\hline Present & Absent & Total \\
& $M(S D)$ &
\end{tabular}

\section{Target}

$\begin{array}{llcc}\text { Alcoholic } & 2.02(0.31) & 3.03(0.77) & 2.53(0.50)^{\mathrm{a}} \\ \text { Non-alcoholic } & 2.21(0.39) & 3.15(0.89) & 2.68(0.60)^{\mathrm{a}} \\ \text { Total } & 2.12(0.30)^{\mathrm{b}} & 3.09(0.79)^{\mathrm{b}} & --\end{array}$

Note: Common sub-scripts denote statistically significant differences, $p<.050$. 
ALCOHOL-RELATED ATTENTIONAL BIAS

Table 3.

Alcohol consumption, subjective craving and drinking motives regressed on alcohol-related $A B$.

\begin{tabular}{|c|c|c|c|c|c|c|c|c|c|c|c|c|c|}
\hline \multirow[b]{3}{*}{ Variable } & & \multirow{2}{*}{\multicolumn{4}{|c|}{$\begin{array}{c}\text { Step 1 } \\
p=.013, \Delta \mathrm{R}=.06^{*}\end{array}$}} & \multirow{2}{*}{\multicolumn{4}{|c|}{$\begin{array}{c}\text { Step } 2 \\
p=.272, \Delta \mathrm{R}^{2}=.06\end{array}$}} & \multirow{2}{*}{\multicolumn{4}{|c|}{$\begin{array}{c}\text { Step 3 } \\
p=.119, \Delta \mathrm{R}^{2}=.09\end{array}$}} \\
\hline & & & & & & & & & & & & & \\
\hline & & $b$ & $95 \% \mathrm{CI}$ & $S E$ & $\beta$ & $b$ & $95 \% \mathrm{CI}$ & $S E$ & $\beta$ & $b$ & $95 \% \mathrm{CI}$ & $S E$ & $\beta$ \\
\hline AUDIT & $\begin{array}{l}\text { Alcohol } \\
\text { consumption }\end{array}$ & .02 & $.003, .03$ & .006 & $.25^{*}$ & .01 & $-.003, .30$ & .007 & .19 & .01 & $-.006, .03$ & .008 & .16 \\
\hline ACQ-SF-R & Craving & & & & & .004 & $-.003, .01$ & .003 & .13 & .005 & $-.002, .01$ & .003 & .16 \\
\hline DMQ-R-SF & Enhancement & & & & & & & & & -.03 & $-.12, .06$ & .04 & -.08 \\
\hline & Social & & & & & & & & & .11 & $.005, .21$ & .05 & $.24 *$ \\
\hline & Coping & & & & & & & & & -.08 & $-.16, .004$ & .04 & -.20 \\
\hline & Conformity & & & & & & & & & -.02 & $-.12, .09$ & .05 & -.04 \\
\hline
\end{tabular}

Note: $95 \%$ CI of $b$. AUDIT = Alcohol Use Disorders Identification Test; ACQ-SF-R = Alcohol Craving Questionnaire Short-Form Revised; DMQ$R-S F=$ Drinking Motives Questionnaire Revised Short-Form. 
ALCOHOL-RELATED ATTENTIONAL BIAS

Table 4.

Matrix of unadjusted regression coefficients ( $\beta$ ) between self-report instruments and VCS task performance.

$\begin{array}{lllllll}\text { 1. } & 2 . & 3 \mathrm{a} . & 3_{\mathrm{b}} & 3_{\mathrm{c} .} & 3_{\mathrm{d} .} & 4 .\end{array}$

1. Alcohol consumption (AUDIT)

2. Subjective craving (ACQ) $.51 * * * \quad--$

3. Enhancement Motives (DMQ) $.45 * * * \quad .32 * * \quad--$

3b. Social Motives $.41 * * * \quad .31 * * \quad .50 * * * \quad--$

$3_{\text {c. }}$ Coping Motives $\begin{array}{lllll}.19 & .26 * * & .12 & .20 * & --\end{array}$

3d. Conformity Motives $\begin{array}{lllll}.49 * * * & .51 * * * & .30 * * & .30 * * & .32 * *\end{array}$

4. Alcohol-related AB (VCS Task) $.25 * \quad .22$ $.13 \quad .27 * * \quad-.09$ $.11 \quad--$

Note: $* * * p<.001, * * p<.010, * p<.050$. 


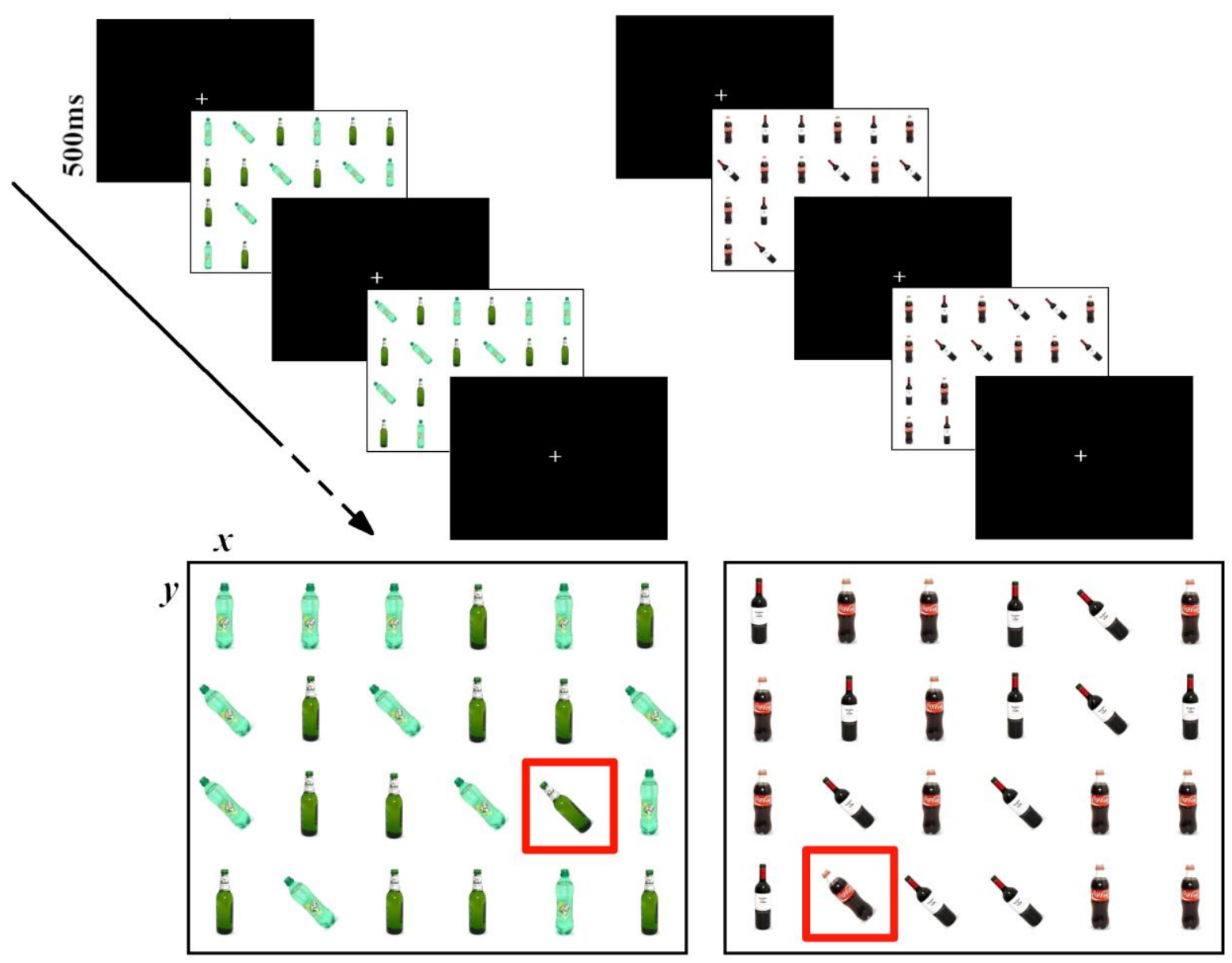

Figure 1. Experimental stimuli. Example arrays presented on one of the two alcoholic (left) and non-alcoholic blocks (right), demonstrating Present trials (targets highlighted red). Responses were recorded as $x-y$ axis locations. 
ALCOHOL-RELATED ATTENTIONAL BIAS

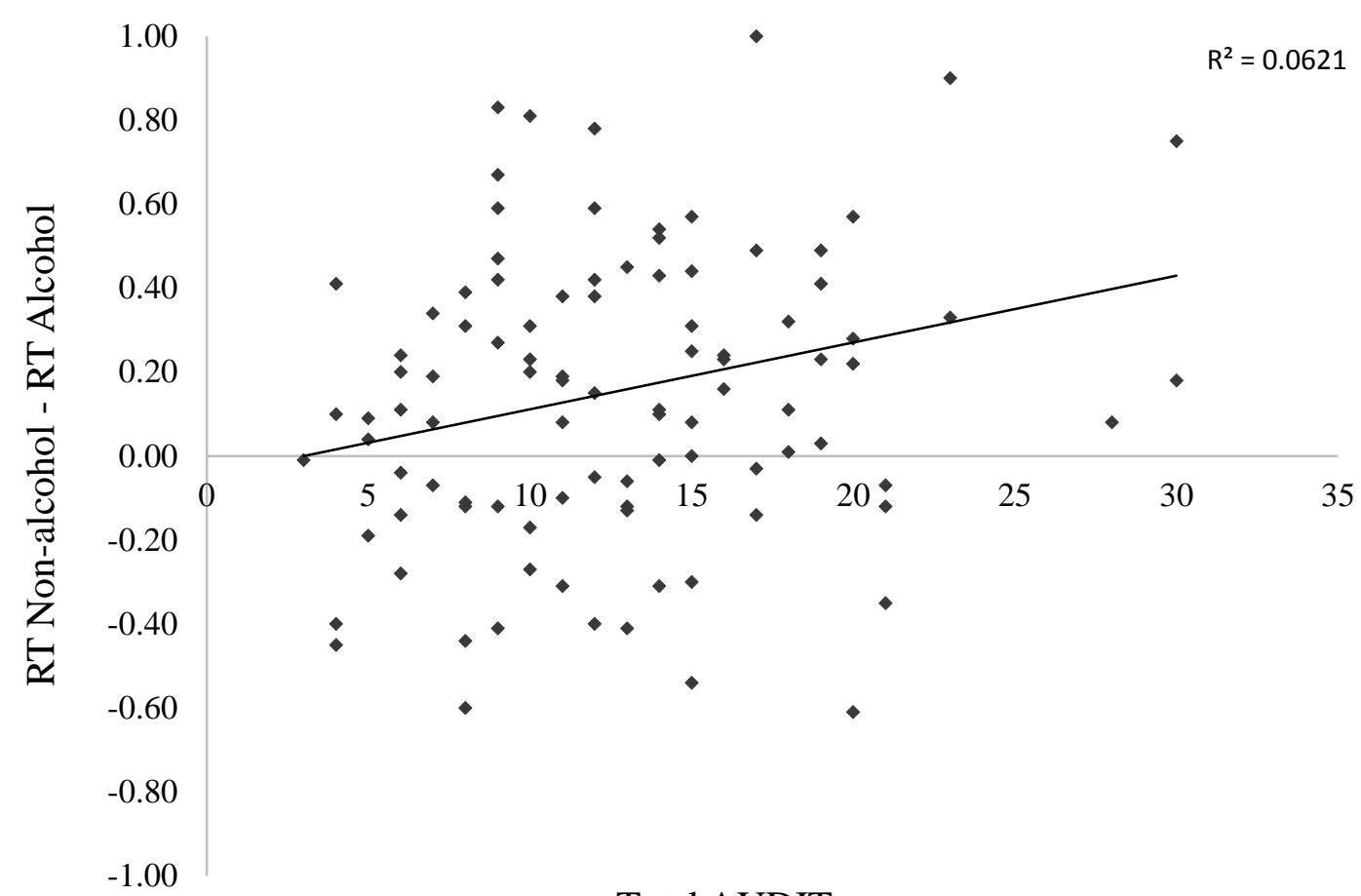

Total AUDIT score

Figure 2. Scatterplot showing the relationship between self-reported alcohol consumption (AUDIT scores) and alcohol-related AB on the VCS task. 